\title{
MORTALIDAD PERINATAL EN INSTITUCIONES HOSPITALARIAS DE BOGOTA*
}

\author{
HOSPITAL SAN JUAN DE DIOS - INSTITUTO MATERNO INFANTIL \\ Dr. Arturo Rodríguez Soto, Dr. Alberto Amaris Mora, \\ Dr. Marcelino Macías, Dr. José David Castillo. \\ HOSPITAL INFANTIL \\ Dr. Jaime Pedraza Gaitán, Dr. Herbert Wagner Cárdenas, \\ Dr. Jaime Enrique Ruiz Acevedo. \\ CLINICA DAVID RESTREPO \\ Dr. Enrique Gutiérrez, Dr. Juan Roa.
}

HOSPITAL MILITAR CENTRAL

Dr. Roberto Páez Vargas, Dr. Reinaldo Heredia.

\section{Objetivos:}

1? Estudiar la Mortalidad Perinatal intrahospitalaria en algunas instituciones de la ciudad de Bogotá y determinar las tasas de esta mortalidad, según las recomendaciones de la $\mathrm{FI}$ GO (6), que define como período perinatal el que comienza cuando el feto pesa 1.000.00 gramos (equivalente a 28 semanas de gestación) y termina cuando el recién nacido ha completado siete días de vida (168 horas).

2. Establecer la relación de mortalidad perinatal y algunos factores de riesgo materno-fetal tales como: edad materna, paridad, peso del producto al nacer.

3. Establecer los posibles factores y causas de defunción en el grupo en estudio.

4. Comparar todos los factores estudiados en las diferentes institucio- nes ya que por las características de éstas, deben ser diferentes.

\section{Material :}

El estudio contempló los siguientes períodos de tiempo y de número de casos:

Instituto Materno Infantil: 25.427 niños nacidos de más de 1.000 gramos y de los cuales 24.791 nacieron vivos y 635 nacieron muertos. Las muertes neonatales en este grupo fueron de 415. Las tasas de mortalidad perinatal básicas se muestran en el Cuadro № 1. Las muertes neonatales fueron 415 y la mortalidad perinatal fue de 1.051 niños.

\footnotetext{
* Este trabajo hace parte del Relato sobre Mortalidad Perinatal presentado en el XI Congreso Colombiano de Obstetricia y Ginecología llevado a cabo en la ciudad de Barranquilla del 2 al 6 de Diciembre de 1975.
} 
De los mortinatos 464 fueron anteparto y 172 intraparto. El tiempo de estudio fue de un año, Septiembre 1? de 1974 a Agosto 31 de 1975.

Clínica David Restrepo: Presenta 10.649 niños nacidos de 1.000 gramos y más, de los cuales 10.547 nacieron vivos y 102 nacieron muertos. Se presentan 95 muertes anteparto $y$ 7 muertes intraparto. Las muertes necnatales fueron 79 . El período de estudio fue de $3 \frac{1}{2}$ años: Enero de 1972 a Junio de 1975.

Hospital Militar Central: Presenta 6.967 niños nacidos de 1.000 gramos y más; 6.874 nacidos vivos y 103 mortinatos. Muertes neonatales 64 . Tiempo de estudio 41/2 años: Enero de 1971 a Junio de 1975.

Hospital Infantil: Presenta 6.615 nacidos de 1.000 gramos y más: 6.554 nacidos vivos y 61 nacidos muertos. Tiempo de estudio, 2 años: Junio de 1973 a Junio de 1975. (Cuadro N: 1 y Gráfica N: 1 ).

En el cuadro y gráfica № 1 se presentän las tasas de mortalidad perinatal para cada institución. Resaltan las cifras elevadas de mortalidad perinatal en el Instituto Materno Infantil que son de $41.3 \times 1.000$ en contraste con las obtenidas en las otras instituciones que son de $24.1 \times 1.000$ para el Hospital Militar, 17.1 x 1.000 para la Clínica David Restrepo y 17 x 1.000 para el Hospital Infantil. Estos datos reflejan las diferentes condiciones socio-económicas de las pacientes y de las Instituciones.

Al desglosar la mortalidad perinatal en mortinatalidad y mortalidad neonatal temprana, se observa que la primerã es de $25 \times 1.000$ en el Instituto Materno Infantil, de $9.6 \mathrm{x}$ 1.000 en la Clínica David Restrepo, de $14.8 \times 1.000$ en el Hospital Militar y de 9.22 en el Hospital Infantil.

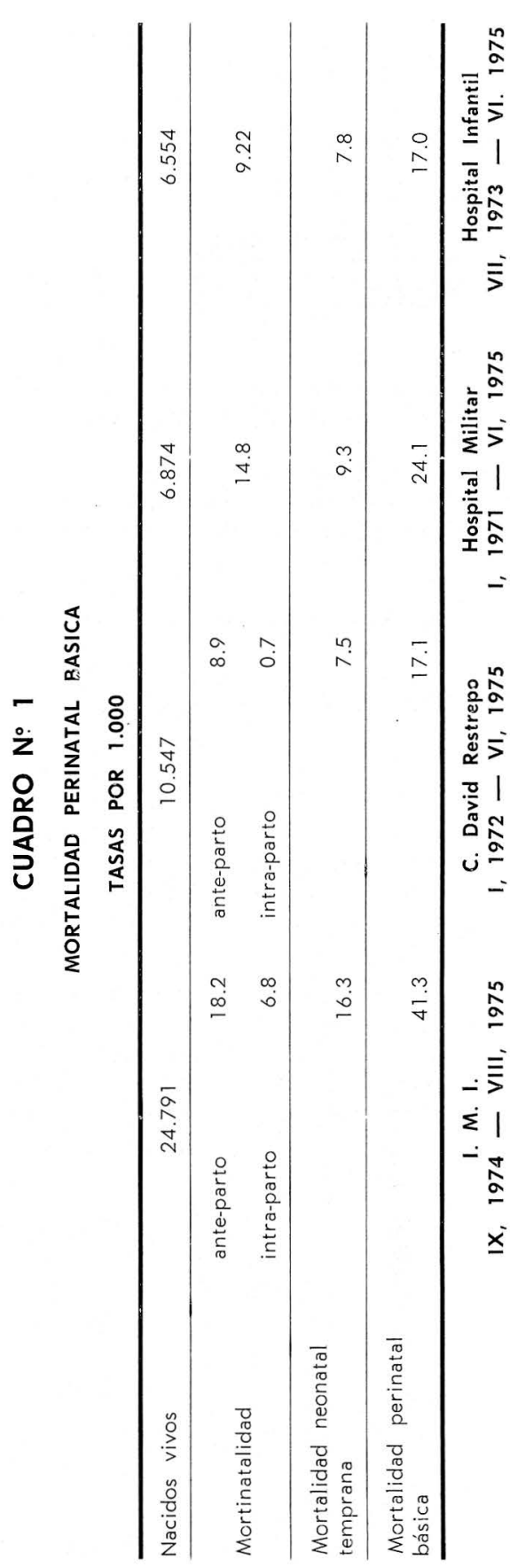




\section{Mortalidad perinatal}

Hospitales de Bogotá

TASAS POR 1.000

H. INFANTIL

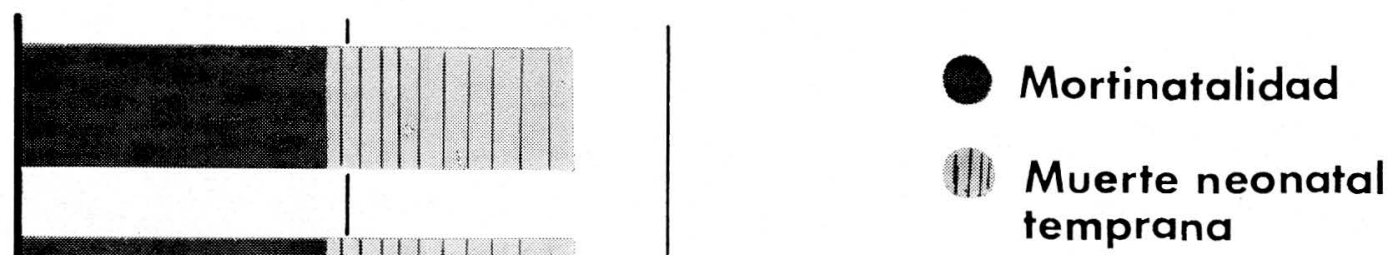

C. DAVID RESTREPO

H. MILITAR

I.MATERNO INFANT.

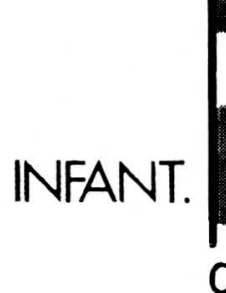

0
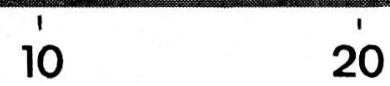

3

40

GRAFICA No 1 
La mortalidad neonatal temprana alcanza cifras de 16.3, 7.5, 9.3 y 7.8 $\times 1.000$ en cada una de estas instituciones.

Considerando que las tasas de mortalidad neonatal temprana en países con adecuados sistemas de protección materno infantil no deben rebasar las cifras de $10 \times 1.000(21,22)$, éstas son muy elevadas en el Instituto Materno Infantil y se encuentran en límites razonables en las otras instituciones.

\section{Frecuencia de niños de bajo peso}

\section{CUADRO N: 2}

FRECUENCIA DE NIÑOS DE BAJO PESO 1975

\begin{tabular}{lr}
\hline INSTITUTO MATERNO INFANTIL & $21,95 \%$ \\
CLINICA DAVID RESTREPO & $10,10 \%$ \\
HOSPITAL INFANTIL & $10,10 \%$ \\
HOSPITAL MILITAR & $7,5 \%$ \\
\hline
\end{tabular}

El peso al nacer sigue siendo a pesar de todas sus limitaciones un parámetro importante para apreciar la salud del recién nacido vivo. Si bien admite variaciones en más o menos con relación a la edad gestacional contada desde la concepción, y distinguiéndose por este hecho niños con crecimiento ponderal acelerado y niños con crecimiento ponderal retardado, siendo estos últimos un grupo importante en el cual hay que diferenciar entre el niño de peso natal insuficiente, calificación que se reserva para los recién nacidos de 2.500 gramos o menos cualquiera que haya sido la duración del embarazo y los niños con retraso de crecimiento hayān nacido antes de término, a término o después del término $(4,7,8$, 1 ú, 17, 18, 23).

Considerando únicamente el factor peso, el Cuadro № 2 muestra la frecuencia de niños de peso insuficiente
Rev. Col. Obst. y Ginec.

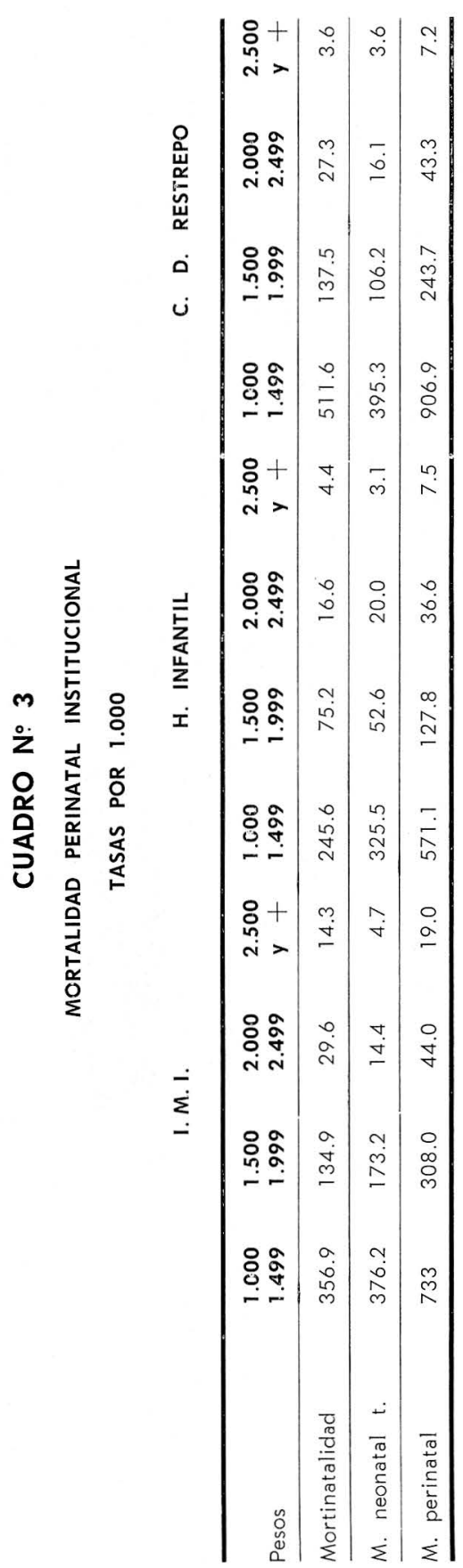




\section{Mortalidad perinatal Institucional-1975}

TASAS POR 1000

I.M.I.

H. INFANTIL

1000

1000

C.D. RESTREPO

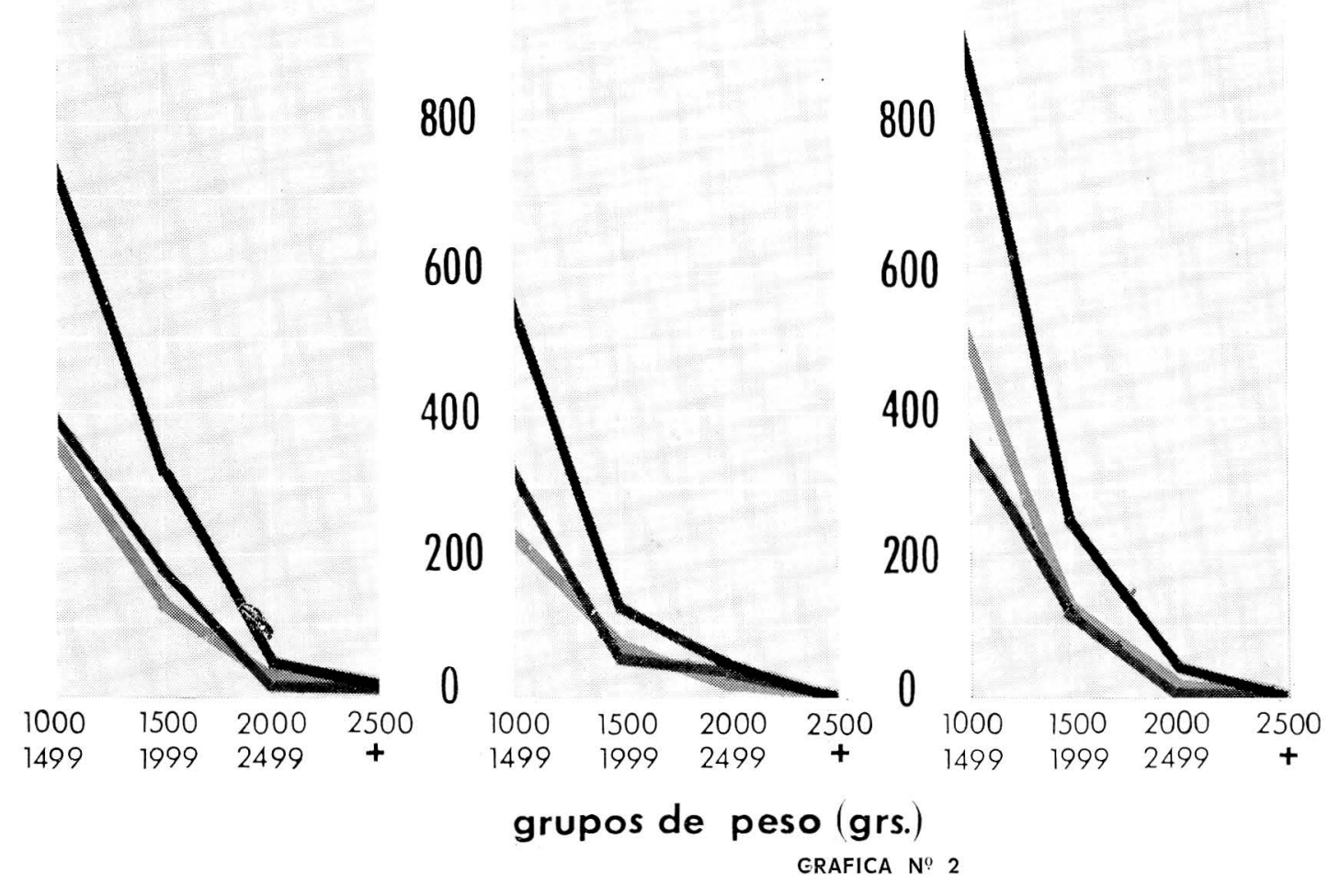

M.perinatal M.neonatal t. Mortinatalidad 
en las cuatro instituciones estudiadas. Se pone en evidencia nuevamente el elevado número de niños de bajo peso al nacer en el Instituto Materno Infantil, ocasionado por la concurrencia en este grupo humano de factores socio-económicos y culturales propios del núcleo de población que concurre a la Institución.

No fue posible en el grupo estudiado correlacionar el peso con la edad gestacional. Como ya se mencionó es importante al efectuar estudios de este tipo, determinar con exactitud el tiempo de gestación ya que el dato de peso aisladamente solo nos indica que el feto no permaneció en el útero el tiempo adecuado o que se desarrolló insuficientemente.

\section{Mortalidad perinatal y grupos de peso:}

\section{(Cuadro № 3 y Gráfica № 2)}

En este cuadro se muestra la mortalidad perinatal por grupos de peso. El valor crítico para el peso al nacer es de 2.500 gramos y ha sido adoptado arbitrariamente por muchas organizaciones, entre ellas la Organización Mundial de la Salud (20), con todas las imperfecciones de este único parámetro, ya que pesos específicos revelan diferentes grados de madurez, pero la facilidad de obtenerlo en grandes grupos de población y las menores posibilidades de error han hecho que se conserve $(13,14)$.

En el presente estudio los grupos de 1.000 a 1.499 gramos y de 1.500 a 1.999 gramos presentan tasas de mortalidad perinatal muy elevadas similares en las instituciones comparadas.

En el grupo de 2.000 a 2.499 gramos hay un descenso notorio con cifras que oscilan entre 36 y 44 × 1.000 . A partir de los 2.500 gramos de mortalidad desciende aun más como es de esperarse; en el Instituto Materno Infantil las tasas son de $19 \times 1.000$ en contraste con los otros hospitales que dan tasas de 7,2 y 7,5 x 1.000.

\section{Mortalidad perinatal y paridad:}

(Gráfico N: 3)

Son numerosos los autores que encuentrān relación muy significativa entre la paridad y la mortalidad perinatal siendo en la gran multípara esta mortalidad muy elevada $(1,3,5$, 9, 12, 19).

En el Instituto Materno Infantil el primer embarazo da una tasa de mortalidad perinatal de $41 \times 1.000$; en el segundo $45 \times 1.000$; en el tercero 18 $x$ 1.000. Después del séptimo embarazo la tasa de mortalidad perinatal es de $88 \times 1.000$.

En el Hospital Militar las tasas pará el primer embarazo son de $18 \mathrm{x}$ 1.000 ; en las grandes multíparas sobrepasan las cifras de $65 \times 1.000$. El mismo fenómeno se observa en la Clínica David Restrepo, es decir un gran incremento de la mortalidad perinatal después del séptimo embarazo.

\section{Edad materna y mortalidad perinatal:} (Gráfico № 3)

Nuestras observaciones están de acuerdo con la mayoría de autores que encuentran que a medida que aumenta la edad de la madre aumenta también el riesgo para el niño, reflejado en aumento de la morbi-mortalidad perinatal. En nuestro estudio encontramos que el menor riesgo está en el grupo de 20 a 24 años y que se incrementa considerablemente después de los 30 años.

\section{Mortalidad perinatal - Causas}

(Cuadro N: 4)

No existe acuerdo unánime para clasificar las causas de mortalidad pe- 


\section{Mortalidad perinatal - 1975}

TASAS POR 1.000

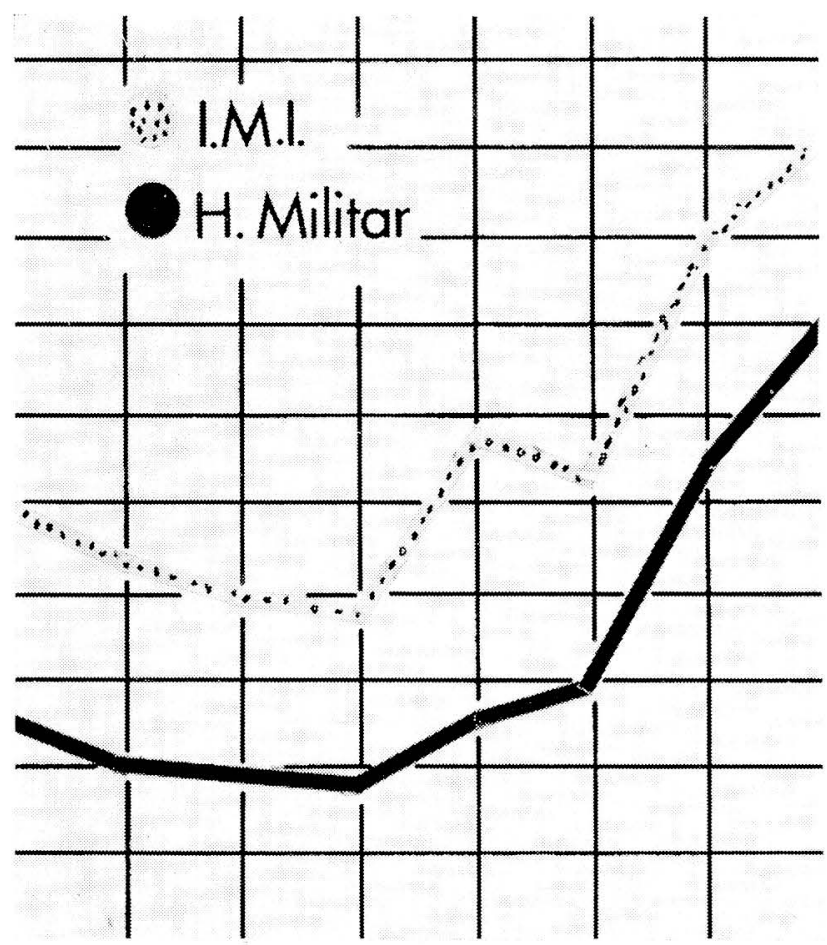

$15.1920 .2425 .2930 .3435 .3940 .44 \quad 45+$ grupos de edad

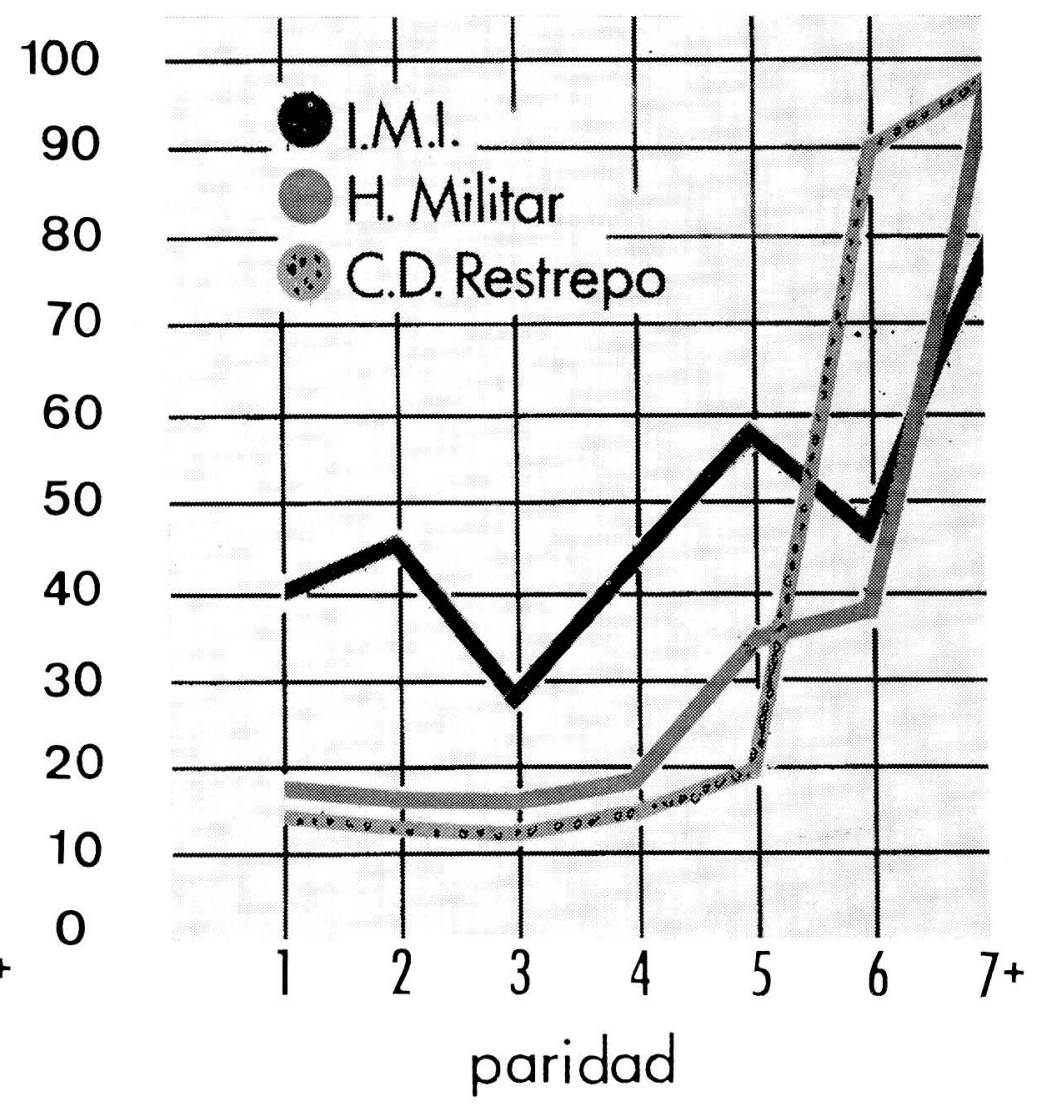

GRAFICA N ${ }^{\circ} 3$ 
rinatal. Steer (16) recomienda clasificarlas en cinco grandes grupos: prematuridad, traumatismo, asfixia, malformaciones y causas desconocidas.

Cuando no se dispone de datos anatomopatológicos, el análisis de las causas de muerte pone de manifiesto una gran variedad de diagnósticos, que lamentablemente no se pueden comprobar.

En el presente estudio hemos clasificado las muertes perinatales en 10 grupos que se muestran en el Cuadro No 4 y que están basadas en la clasificación internacional de enfermedades.

\section{CUADRO N 4}

MORTALIDAD PERINATAL - GRUPOS DE CAUSAS

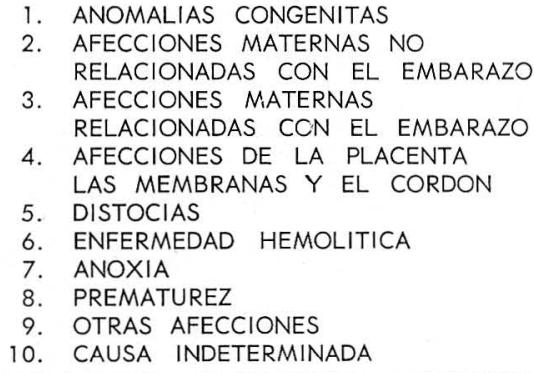

Se han analizado separadamente las muertes anteparto, intraparto $y$ neonatales tempranas ya que los factores etiológicos son diferentes para cada grupo.

Hemos comparado las causas de muerte en el Instituto Materno Infantil y en la Clínica David Restrepo.

\section{Mortalidad anteparto}

\section{(Cuadro № 5)}

El número de muertes anteparto en el Instituto Materno Infantil en el año de estudio fue de 464 que corresponden al 72.95 de la mortinatalidad.

En la Clínica David Restrepo en el período de estudio (Enero de 1972, Junio de 1973) el número de muertes anteparto fue de 95 que corres-

\author{
Diabetes, E. cardiovasculares, \\ E. renales, sifilis, toxoplasmosis. \\ Toxemia, pielitis, pielonefritis, \\ corioamniotitis, hidramnios, E. múltiple. \\ Ruptura membranas, Placenta previa, \\ abruptio. nudos, circulares, procidencias.
}

ponden a $93.13 \%$ de la mortinatalidad.

Las posibles causas de muerte anteparto en el Instituto Materno Infantil fueron la toxemia gravídica en un $10.5 \%$; las afecciones del cordón y la placenta en un $10.9 \%$; las afecciones cardiovasculares y renales en un $5.4 \%$ y las anomalías congénitas incompatibles con la vida en un $4.4 \%$. En el grupo de causas indeterminaclas se anota un $68.8 \%$.

En la Clínica David Restrepo la toxemia gravídica constituyó el $21.05 \%$ las enfermedades de la placenta y el cordón el $26.35 \%$; l as enfermedades cardiovasculares y renales $22.1 \%$ y las anomalías congénitas un $2.1 \%$. En el capítulo de causas indeterminadas se encuentra un $27.35 \%$. 
CUADRO N

CAUSAS DE MORTALIDAD ANTEPARTO - 1975

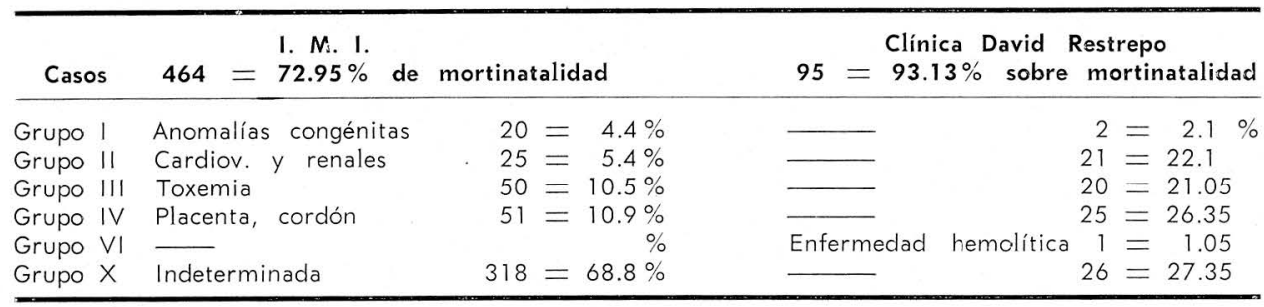

\section{Mortalidad intraparto}

(Cuadro N: 6)

En el Instituto Materno Infantil se presentaron 172 casos de muerte intraparto que corresponden al $28.05 \%$ de la mortinatalidad.

En la Clínica David Restrepo se presentaron siete casos que corres- ponden al $6.87 \%$ de la mortinatalidad.

Al efectuar el estudio de la mortinatalidad intraparto se determinó que la mayoría de las muertes (67\%) ocurrieron después de cumplidas las 37 semanas de gestación y cuando el feto pesaba más de 2.500 gramos.

\section{CUADRO № 6}

CAUSAS DE MORTALIDAD INTRAPARTO - 1975

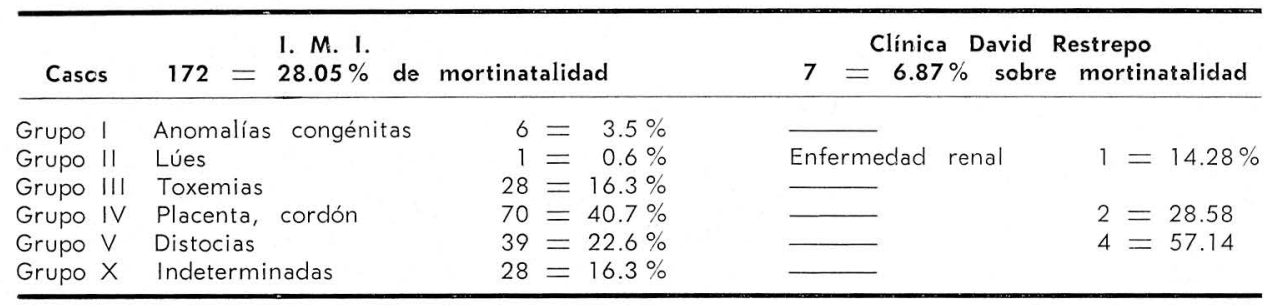

El perfeccionamiento de las técnicas obstétricas, la identificación pre$\mathrm{coz}$ de los fetos de alto riesgo y su cuidadosa vigilancia en el trabajo de parto, deben determinar una disminución de la mortalidad intraparto, la cual es en su mayor parte una mortalidad prevenible.

En este grupo las afecciones del cordón y la placenta como causas de muerte representa en el Instituto $\mathrm{Ma}$ terno Infantil el $40.7 \%$ y en la Clí- nica David Restrepo el $28.58 \%$. Le siguen como causas de muerte intraparto las clasificadas como toxemia gravídica con un $16.3 \%$ en el Instituto Materno Infantil. En la Clínica David Restrepo no se encuentra ninguna muerte intraparto debida a la toxemia gravídica. Las complicaciones de las distocias dan un $22.6 \%$ en el Instituto Materno Infantil y $57.14 \%$ en la Clínica David Restrepo. La enfermedad renal da en la Clínica David Restrepo un $14.28 \%$. Las 
anomalías congénitas se encuentran en un $3.5 \%$ en el Instituto Materno Infantil y las causas indeterminadas en un $16.3 \%$.

\section{Mortalidad neonatal temprana}

\section{(Cuadro N: 7)}

Al estudiar la mortalidad neonatal temprana, se encuentra como posi- bles factores causales los relacionados con el trauma obstétrico, los defectos de ventilación pulmonar y las infecciones (15). En estudios de mortalidad neonatal efectuados por Poter y Bundesen $(2,11)$ se encontró que muchos de los niños cuya muerte se achacaba a prematurez mostrabän extensa zona de atelectasia pulmonar.

\section{CUADRO N: 7}

CAUSAS DE MORTALIDAD NEONATAL TEM:PRANA

\begin{tabular}{|c|c|c|c|c|c|c|}
\hline & \multicolumn{3}{|c|}{ I.M.I. } & \multirow{2}{*}{$\begin{array}{l}\text { C. } \\
13\end{array}$} & \multirow{2}{*}{ D. } & \multirow{2}{*}{$\begin{array}{l}\text { Restrepo* } \\
=\quad 16.45 \%\end{array}$} \\
\hline Anomalías congénitas .. & 18 & $=$ & $4.3 \%$ & & & \\
\hline 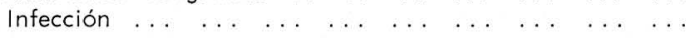 & 90 & $=$ & 22.0 & 1 & $=$ & $=1.25$ \\
\hline $\begin{array}{llllllllll}\text { Anoxia } & \ldots & \ldots & \ldots & \ldots & \ldots & \ldots & \ldots & \ldots & \ldots\end{array}$ & 174 & $=$ & 42.0 & 17 & $=$ & 21.50 \\
\hline $\begin{array}{llllllll}\ldots & \ldots & \ldots & \ldots & \ldots & \ldots & \ldots\end{array}$ & 46 & $=$ & 11.0 & 3 & $=$ & 3.80 \\
\hline Síndrome dificultad respiratoria $\ldots \ldots \begin{array}{lllll}\ldots & \ldots & \ldots & \ldots\end{array}$ & 62 & $=$ & 15.0 & 37 & $=$ & 46.85 \\
\hline Enfermedad hemolítica $\ldots \ldots$. . & 6 & $=$ & 1.4 & 6 & $=$ & 7.60 \\
\hline 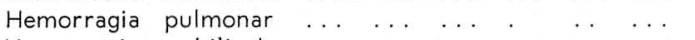 & 2 & $=$ & 0.4 & & - & \\
\hline 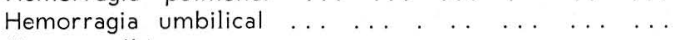 & 1 & $=$ & 0.2 & & - & \\
\hline $\begin{array}{lllllllll}\text { Muerte súbita } & \ldots & \ldots & \ldots & \ldots & \ldots & \ldots & \ldots & \ldots\end{array}$ & 2 & $=$ & 0.4 & & - & \\
\hline 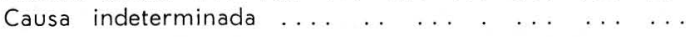 & 14 & $=$ & 3.3 & 2 & $=$ & 2.55 \\
\hline $\begin{array}{c}\text { TOTAL } \\
* \text { Autopsias } \\
69.6 \%\end{array}$ & 415 & $=$ & $100 \%$ & 79 & $=$ & $=100$ \\
\hline
\end{tabular}

Desde hace 15 años la membrana hialina y la ventilación pulmonar anormal principian a aparecer con frecuencia como causas de muerte temprana después del nacimiento. La membrana hialina a partir de 1958 empieza a ser incluída en la categoría de enfermedades definidas y peculiares del período neonatal temprano (11).

Los criterios modernos exigen una atención especializada del feto que corre riesgo alto en el período prenatal, en el parto y en el período neonatal temprano. Solo así se podrán disminuir las tasas de mortalidad neonatal temprana.

\section{Causas de muerte neonatal temprana}

Solo fue posible comparar el Instituto Materno Infantil y la Clínica
David Restrepo. En el Cuadro N? 7 se agrupan las principales causas de mortalidad neonatal temprana. La anoxia fue la causa prevalente en un $42 \%$ en el I.M.I. y en un $21.5 \%$ en la Clínica David Restrepo. La infección mostró cifras de $22 \%$ en el I.M.I. y de $1.25 \%$ en la Clínica David Restrepo. El signo de dificultad respiratoria dá cifras de $15 \%$ en el I.M.I. y de $46.85 \%$ en la Clínica David Restrepo. Las anomalías congénitas alcanzaron el $4.3 \%$ en el I.M.I. y el $16.45 \%$ en la Clínica David Restrepo.

El hecho de que causas distintas a las änomalías congénitas alcancen un elevado predominio en la muerte neonatal temprana pone de presente el predominio de factores socio-econó- 
micos del grupo humano y de las instituciones.

Es de esperar que estas cifras disminuyan significativamente cuando mejoren las condiciones de vida de este grupo etáreo.

\section{Recomendaciones}

El grupo de médicos que realizó el estudio de mortalidad perinatal en instituciones hospitalarias de Bogotá, al analizar las cifras de esta mortalidad, los factores que la incremen$\tan$ y sus causas, ha planteado las recomendaciones que se transcriben en el Cuadro № 8, las cuales al ponerse en práctica, pueden lograr una reducción significativa de la mortalidad y la morbilidad perinatales.

\title{
CUADRO NN 8
}

\author{
LA MORTALIDAD PERINATAL ESTA INFLUENCIADA \\ POR LAS CONDICIONES PRENATALES Y LAS CIRCUNSTANCIAS DEL PARTO.
}

\section{RECOMENDACIONES}

\begin{abstract}
Organización de asistencia
prenatal y postnatal.

Niveles de atención

según riesgo obstétrico.

Vigilancia permanente del

feto durante el parto.

Unidades de cuidado intensivo

para nacidos de alto riesgo.

Creación de Comités de estudio

permanente de mortalidad perinatal.

Análisis de muertes prevenibles.

Tabulación cruzada de peso al nacer y edad gestacional.
\end{abstract}

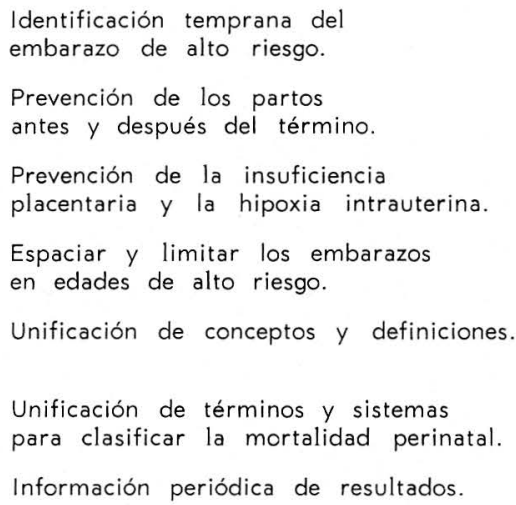

Políticas adecuadas de protección materno-infantil a nivel local y nacional.

\section{Resumen y Conclusiones}

Se presenta un estudio de mortalidad perinatal intrahospitalaria en cuatro instituciones de la ciudad de Bogotá, las cuales representan diferentes grupos humanos con condiciones socioeconómicas y culturales diversas.

$2^{\circ}$. Las tasas de mortalidad perinatal obtenidas son: Instituto Materno Infantil $41.3 \times 1.000$; Hospital Mili$\operatorname{tar} 24,1 \times 1.000$; Clínica David Res- trepo $17,1 \times 1.000$ y Hospital Infantil $17 \times 1.000$.

3: Las tasas de mortinatalidad son superiores a la mortalidad neonatal temprana en las cuatro instituciones.

4. La frecuencia de nacimientos de niños de bajo peso es de $21,95 \%$ en el Instituto Materno Infantil; $10,1 \%$ en el Hospital Infantil y de $7,5 \%$ en el Hospital Militar.

5: En todas las instituciones estudiadas se encontraron cifras de mor- 
talidad perinatal muy elevadas en niños con pesos inferiores a $1.500 \mathrm{gra}$ mos.

6. Existe una relación significativa entre mortalidad perinatal, edad materna y paridad. Mujeres con más de 30 años o con más de 5 gestaciones muestran incremento notorio de la mortalidad perinatal.

7: Las causas de mortalidad perinatal se estudiaron en el anteparto, en el intraparto $y$ en el período neonatal temprano.

En el anteparto las más frecuentes fueron: complicaciones de la toxemia gravídica, afecciones del cordón y la placenta y las anomalías congénitas incompatibles con la vida.

En el período intraparto se encuentran las afecciones del cordón y la placenta, la toxemia gravídica con sus repercusiones sobre el feto y las complicaciones ocasionadas por las distocias.

En el período neonatal temprano las cauusas prevalentes de muerte se relacionan con las afecciones causantes de anoxia y con las infecciones.

8: Se plantean algunas recomendaciones para disminuir las tasas de mortalidad perinatal en el país tales como: identificación temprana del embarazo de alto riesgo, niveles de atención según riesgo obstétrico, unidades de cuidado intensivo materno fetal, comités permanentes para estudio de mortalidad, unificación de términos y criterios para determinar políticás adecuadas de protección maternoinfantil a nivel local y nacional.

\section{Summary and Conclusions}

1. An in hospital perinatal mortality study in four institutions in Bogotá is put forward. These institutions represent different human groups with diverse social, economic and cultural conditions.

2. The perinatal mortality rates obtained are the following: Instituto Materno Infantil, $41.3 \times 1.000$; Hospital Militar, 24.1 x 1.000; Clínica David Restrepo, $17.1 \times 1.000$; and Hospital Infantil, $17 \times 1.000$.

3. The stillbirth rates are higher than the early neonatal mortality rates in the four institutions.

4. The frequency of lowbirth weight infants is 21.95 per cent at the Instituto Materno Infantil; 10.1 per cent at the Hospital Infantil and 7.5 per cent at the Hospital Militar.

5. In all the institutions under study very high perinatal mortality figures on infants under $1.500 \mathrm{grm}$. were found.

6. There is a significative relationship between perinatal mortality, age of the mother and parity. Women over 30 years or with more than five gestations show a notorious perinatal mortality increase.

7. The causes of perinatal mortality were studied during the antepartum, intrapartum and neonatal periods.

The more frequent causes during the antepartum were: complications of the toxemia of pregnancy, umbilical cord and placent diseases and congenital anomalies incompatible with life.

During the intrapartum, umbilical cord and placent disease are found, as well as the toxemia of pregnancy with its repercussions over the fetus and the complications caused by dystocias.

During the early neonatal period, the prevailing death causes are rela- 
ted to diseases producing anoxia and infectious diseases.

8. Some recommendations are made to reduce the perinatal mortality rates country, such as: early identification of high risk pregnancy, levels of care according to obstetric risk, intensive care units for the maternal fetus, permanent commissions for the study of mortality, unifications of terms and criteria to determine adequate policies for mother and child protection at the local and national levels.

\section{BIBLIOGRAFIA}

1 BRICEÑO, R. T., RIVAS, H., y GRIECO, M. Mortalidad perinatal. Análisis de 1.148 cascs. Rev. de Obst. y Ginec. de Venezuela. 31 : 193, 1971.

1 BUNDESEN, H. N., POTTER, E. L.. FISBEIN, W. I., BAUER, F. C. and PLOTZKE, G. L. : Progress in the prevention of meedless neonatal deaths. Annual report of the Chicago Health department, 1951.

3 DUARTE, A., ZUÑIGA, R., CORONEL, JOSE, y BUSTAMANTE, G.: Mortalidad Perinatal en el Hospital San Juan de Dios, Cúcuta. Rev. Col. Obst. y Ginec. 15: 319, 1964.

4 ERHARDT, C. L., JOSHI, G. B., NELSON, F. G., KROLL, B. H. and WEINER, L.: Influence of wieght and gestation of perinatal mortality by ethnic groups. Amer. J. Publ.

Hlth. 54: 1841, 1964.

5 ESCOBAR, J., GOMEZ, A., QUINTERO, C. : Mortalidad perinatal. Rev. Col. Obst. y Ginec. 15: 296, 1964.

6 F.I.G.O. News definition of terms in human reproduction. International Journal of Gynecology \& Obstetrics. 12: 30, 1974.

7 GRUENWALD. P.: Infants of low birth weigth among 5.000 deliveries. Pediatrics. $34: 157,1964$.

8 LESINSKI, J.: Relationship between length of gestation, birth weigth and certain other factors. Statistical Study Bull. Wld. HIth. Org. 26: 183, 1962.

9 MAZZOCO de RUSSANT, N.: Mortalidad perinatal. Obstetricia y Ginecología Latinoamericanas. 31: 269, 1973.
10 PAGE, E. W.: Pathogenesis and prophylasix of low birth weights. Clin. Obstet. Gynec. 13: $79,1970$.

11 POTTER, E. L.: Pathology of the fetus and the newborn. Chicago: Year book Publishers. 1952.

12 PUFFER, R., y SERRANO C. V.: Características de la mortalidad en la niñez. OPS. Publicación científica 262, Washington, D.C. 1973.

13 RCSA, F., and RESNICK. L.: Birth weight and perinatal mortality in the American Indian. Am. J. Obst. Gyn. 55: 972, 1965.

14 SCHELESINGER, E. R., and ALLAWAY, N. C. : The combined effect of birth weigth and length of gestation on neonatal mortality among single premature births. Pediatrics. 15: $698,1955$.

15 SCHELESINGER, E. R.: Infant and perinatal mortality. Am. J. Pub. Health. 57: 1.715, 1967.

16 STEER, C. M. and MOORE, J. G.: Course of perinatal mortality: A review of etiologic factors in the Sloane Hospital. 1.888, 1967. Obstet. and Gynec. 34: 113, 1969.

17 TERRIS, M. and GOLD, E. M. : Epidemiologic study of prematurity. Amer. J. Obstet Gynec. 103: 358, 1968.

18 VAN DENBERG, B. J. and YERUSHALMY. J.: Relationship of the rate of intrauterine growth of infants of low birthweight to mortality, morbility and congenital anomalies. J. Pediat. 69: 531, 1966.

19 VASQUEZ, J., OVIES, A., GALINDO, C., CANDEBAT, Z., y HEREDIA, M. : Informe preliminar del estudio de la mortalidad perinatal en la Provincia de La Habana. Rev. Cub. Ped. 44: 53, 1972.

20 World Health Organization, Expert committee on maternal and child Health. Public Health Aspects of low Birth weight. Wld. Hlth. Org. techn. Rep. Ser. No 217, Geneva, 1961.

21 World Health Organization. Epidemiological and Vital Statistics Report. Vo!. 19, No 3 1966, pg. 100.

22 World Health Organization. World Health Statistics Report. Vol. 22, No 1. 1969, pg. 46 . 